INPLASY

PROTOCOL

To cite: Li et al. Regional citrate versus heparin anticoagulation for CRRT in critically ill patients: a metaanalysis of RCTS. Inplasy protocol 2021120093. doi: 10.37766/inplasy2021.12.0093

Received: 21 December 2021

Published: 21 December 2021

Corresponding author:

Peirong Zhang

zprtxzz@163.com

Author Affiliation:

Weifang Medical University.

Support: No.

Review Stage at time of this submission: Data analysis Completed but not published.

Conflicts of interest:

None declared.

\section{Regional citrate versus heparin anticoagulation for CRRT in critically ill patients: a meta-analysis of RCTS}

\author{
Li, R'; Gao, X²; Zhou, T33 Li, YJ4; Wang, JH55; Zhang, PR6.
}

Review question / Objective: Compare the safety, advantages, and disadvantages of regional citrate versus heparin anticoagulation for CRRT in critically ill patients.

Condition being studied: Continuous renal replacement therapy (CRRT) is commonly used for the treatment of critically ill patients with acute kidney injury. In CRRT, effective cardiopulmonary bypass anticoagulation must be performed to prevent clotting of the extracorporeal line or filter and maintain the performance of the filter. Several studies have shown that a variety of anticoagulant treatments have benefits on circuit loss compared with treatment without anticoagulant use. Citrate and heparin are two of the most widely used anticoagulant regimens in the world.Heparin can increase the risk of bleeding and even cause complications such as heparin-induced thrombocytopenia (HIT). Citrate anticoagulation is an ideal method for CRRT and can be used safely in patients with coagulation disorders; however, it may cause hypocalcemia, metabolic alkalosis, and other metabolic disorder symptoms. A large number of studies have compared the advantages and disadvantages and the safety of anticoagulation between citrate and heparin, but these findings remain controversial.

INPLASY registration number: This protocol was registered with the International Platform of Registered Systematic Review and Meta-Analysis Protocols (INPLASY) on 21 December 2021 and was last updated on 21 December 2021 (registration number INPLASY2021120093).

\section{INTRODUCTION}

Review question / Objective: compare the safety, advantages, and disadvantages of regional citrate versus heparin anticoagulation for CRRT in critically ill patients

Condition being studied: Continuous renal replacement therapy (CRRT) is commonly 
used for the treatment of critically ill patients with acute kidney injury. In CRRT, effective cardiopulmonary bypass anticoagulation must be performed to prevent clotting of the extracorporeal line or filter and maintain the performance of the filter. Several studies have shown that a variety of anticoagulant treatments have benefits on circuit loss compared with treatment without anticoagulant use. Citrate and heparin are two of the most widely used anticoagulant regimens in the world.Heparin can increase the risk of bleeding and even cause complications such as heparin-induced thrombocytopenia (HIT). Citrate anticoagulation is an ideal method for CRRT and can be used safely in patients with coagulation disorders; however, it may cause hypocalcemia, metabolic alkalosis, and other metabolic disorder symptoms. A large number of studies have compared the advantages and disadvantages and the safety of anticoagulation between citrate and heparin, but these findings remain controversial.

\section{METHODS}

Participant or population: Critically ill adult patients.

Intervention: Citrate anticoagulation.

Comparator: Heparin anticoagulation

Study designs to be included: RCTs.

Eligibility criteria: Inclusion criteria: RCT studies. The intervention was anticoagulation with citrate or heparin. Study subjects were adults (aged $>18$ years).Exclusion criteria: Patients with liver failure or hemorrhagic disease, duplicate reports, and poor quality studies. The required data cannot be extracted from the published results.

Information sources: Relevant studies in PubMed, Embase, and Cochrane Library databases from database inception until September 2021, including RCTs comparing anticoagulation with citrate versus heparin in CRRT.

Main outcome(s): Mortality and filter life.

Additional outcome(s): circuit loss, bleeding, heparin-induced thrombocytopenia (HIT), metabolic alkalosis, hypocalcemia, and transfusion events

Quality assessment / Risk of bias analysis: The quality of included studies was assessed by using standard criteria: random sequence generation, allocation concealment, blinding of participants and personnel, blinding of outcome assessment, incomplete outcome data, selective reporting and other bias. Publication bias was evaluated using funnel plots.

Strategy of data synthesis: Statistical analyses were conducted using the RevMan5.4 software. Relative risk and mean difference were used as effect analysis statistics for dichotomous and continuous variables, respectively, and 95\% confidence intervals were calculated. The heterogeneity among the included studies was analyzed using the $x 2$ test and quantitatively judged using the 12 value. If there was no heterogeneity among the studies $(P \geq 0.1,12 \leq 50 \%)$, the fixed-effect model was used for analysis. If there was heterogeneity among the studies $(P<0.1$ 、

$12>50 \%$ ), the random effect model was used for analysis. Publication bias was evaluated using funnel plots. $P<0.05$ was considered to be statistically significant.

Subgroup analysis: Conducted a subgroup analysis of patients treated with $\mathrm{CVVH}$ / CVVHDF, predilution, or postdilution.

Sensitivity analysis: Sensitivity analyses were performed by sequentially excluding the study.

Country(ies) involved: China.

Keywords: continuous renal replacement therapy; Randomized controlled trials; 
meta-analysis; Heparin-induced thrombocytopenia.

Contributions of each author:

Author 1 - Rui Li.

Email: liruiyoo@163.com

Author 2 - Xiang Gao.

Author 3 - Tao Zhou.

Author 4 - Yunjie Li.

Author 5 - Jianhui Wang.

Author 6 - Peirong Zhang.

Email: zprtxzz@163.com 